\title{
Outcome of Strabismus in Children with Cataracts
}

\author{
Lingkun Kong, Bradley L. Shoss, Karen A. Alvarez, Kimberly G. Yen
}

Department of Ophthalmology, Cullen Eye Institute, Baylor College of Medicine, Houston, USA.

Email: lkong@bcm.edu

Received August $6^{\text {th }}, 2013$; revised September $6^{\text {th }}, 2013$; accepted October $6^{\text {th }}, 2013$

Copyright (C) 2013 Lingkun Kong et al. This is an open access article distributed under the Creative Commons Attribution License, which permits unrestricted use, distribution, and reproduction in any medium, provided the original work is properly cited.

\begin{abstract}
We characterized the outcome of strabismus in 54 pediatric patients with cataracts. Patients were less than 18 years of age at the time of undergoing cataract extraction with or without intraocular lens (IOL) implantation. Of 54 patients, 24 (44\%) were male; $30(56 \%)$ were female. $36(67 \%)$ had congenital cataract; $18(33 \%)$ had acquired cataract. Average age at cataract surgery was 40.39 months (SD: 38.82 ; range: 2 - 156). All patients had strabismus at the time of diagnosis of the cataracts. One year after cataract surgery, 39 (72\%) patients had resolution or improvement of strabismus without surgery at an average of 3.8 months (SD: 3.6; range 0 to 12 months); 15 (28\%) of patients required strabismus surgery. No association was found between resolution of strabismus, gender, the presence of congenital or acquired cataract, unilateral or bilateral cataract, duration of cataract, and the presence of IOL or aphakia. Strabismus was more likely to resolve without surgical intervention if the child was older at the time of diagnosis of cataract. The predicted value of resolution at 50 months of age is $70 \%$ and increases with age.
\end{abstract}

Keywords: Strabismus; Cataract; Aphakia

\section{Introduction}

Pediatric cataracts remain a significant contributor to vision loss in children worldwide [1,2]. Strabismus has been found to occur more frequently in patients with developmental cataracts compared to that of the general population. In the general population, the incidence of strabismus is reported to be $2 \%$ to $5 \%[3,4]$. In a study of 113 pediatric patients with developmental cataracts by Magli et al. (2008) [5], however, strabismus was present in $34 \%$ of the patients prior to cataract surgery.

There is little documented evidence in the literature regarding the outcomes of strabismus after cataract surgery. This paper aims to characterize the outcome of strabismus in pediatric patients who present with cataracts and strabismus.

\section{Patients and Method}

This retrospective study was approved by the Institutional Review Board. Medical records were reviewed of all patients less than 18 years of age who underwent cataract extraction with or without IOL implantation between January 2002 and December 2010. Patients were excluded if they did not have reported strabismus or if strabismus measurements were not performed before or after the cataract surgery. Data collected included the patient's age at surgery, gender, nature of the strabismus (intermittent versus (vs.) constant and esotropia vs. exotropia), nature of the cataract(s) (congenital vs. acquired and unilateral vs. bilateral), presence of amblyopia, and resolution of the strabismus after surgery. This sample was then divided into 2 subgroups: those whose strabismus resolved after cataract surgery and those whose strabismus did not.

Strabismus was recorded as significant if it was greater than 10 prism dioptor (PD) on physical exam using Krimsky technique or prism alternate cover test at near. All surgeries were performed by a single surgeon (KGY) using the standard technique that has been described in the literature [6]. The two subgroups were compared using a chi-squared test for significance. Data were analyzed in a logistic regression model which included the following variables: gender, esotropia vs. exotropia, intermittent vs. constant deviation, amblyopia vs. no amblyopia, congenital vs. acquired, IOL vs. aphakic, and bilateral vs. unilateral cataracts.

\section{Results}

\subsection{Characteristics of Patients}

After reviewing the selected charts, 54 patients with both 
cataract and strabismus at the time of diagnosis of the cataract(s) were included in this study. The characteristics of the eyes are summarized in Table 1. Of 54 patients, $24(44 \%)$ were male and $30(56 \%)$ were female. $36(67 \%)$ had congenital cataract and 18 (33\%) had acquired cataract. The average age at diagnosis of cataract was 37.3 months (SD: 37.2; range 1 - 156) and average age at cataract surgery was 40.39 months (SD: 38.82; range: 2 - 156).

Thirty two (59\%) patients had intermittent strabismus and $22(41 \%)$ had constant strabismus. Three (5\%) had a vertical form of strabismus (1 had DVD, 1 had hypertropia, and 1 had both XT and hyperopia). Within one year after cataract surgery, 34 (63\%) patients had resolution of their strabismus without strabismus surgery at an average time of 3.8 months after the cataract surgery (SD: 3.6; range 0 to 12 months). 5 (9\%) patients had improvement of strabismus and did not require surgery because their angle was less than 10 PD. In 15 (28\%) pa-

Table 1. Characteristics of patients with strabismus and cataract.

\begin{tabular}{|c|c|c|}
\hline Variables & No. & $\%$ \\
\hline \multicolumn{3}{|l|}{ Gender } \\
\hline Male & 24 & 44 \\
\hline Female & 30 & 56 \\
\hline \multicolumn{3}{|l|}{ Character of cataract } \\
\hline Congenital & 36 & 67 \\
\hline Acquired & 18 & 33 \\
\hline \multicolumn{3}{|l|}{ Eye(s) involved } \\
\hline Unilateral & 40 & 74 \\
\hline Bilateral & 14 & 26 \\
\hline \multicolumn{3}{|l|}{ Types of deviation } \\
\hline Intermittent & 23 & 43 \\
\hline Constant & 28 & 52 \\
\hline \multicolumn{3}{|l|}{ Resolution } \\
\hline Yes & 34 & 63 \\
\hline No & 15 & 28 \\
\hline Improved & 5 & 9 \\
\hline \multicolumn{3}{|l|}{ Amblyopia } \\
\hline Yes & 41 & 76 \\
\hline No & 13 & 24 \\
\hline \multicolumn{3}{|l|}{ Types of strabismus } \\
\hline ET & 23 & 44 \\
\hline XT & 29 & 56 \\
\hline \multicolumn{3}{|c|}{ Intraocular lens implantation } \\
\hline Yes & 41 & 76 \\
\hline No & 13 & 24 \\
\hline
\end{tabular}

ET: esotropia; XT: exotropia. tients, the strabismus did not improve with cataract surgery alone and the patients required strabismus surgery. At their final visit, all of these patients had an angle of 10 PD or less after strabismus surgery.

\subsection{Risk Factors for Amblyopia}

Of the 54 patients, 17 (31\%) were able to be evaluated using HOTV testing; mean vision was 1.15 LogMar (SD, $0.35)$ before surgery and 0.67 LogMar (SD, 0.41) after the surgery in these eyes. The $37(69 \%)$ other patients could not perform visual acuity due to either young age or lack of cooperation and were assessed as no fix and follow or blink to light, blink to light only, or fix and follow. The patients were divided into three groups: 1) improved vision (for example, an improvement in LogMar acuity; improvement from blink to light only preoperatively to fix and follow postoperatively; or improvement from no fix and follow to fix and follow after surgery) 2) vision unchanged; and 3) vision decreased. Using these criteria, $41(76 \%)$ patients had vision improvement and 13 (24\%) patients had no vision change. Logistic regression test showed that the improvement of vision has significant contribution to the resolution of strabismus $(p=0.015)$.

The presence of amblyopia was correlated with age at cataract diagnosis and surgery $(\mathrm{r}=0.33, p=0.0138)$, and showed that patients without amblyopia were likely to be older at the time of cataract diagnosis and surgery. The presence of amblyopia was correlated with the presence of a unilateral cataract $(p=0.002)$, but was not related to the presence of IOL or aphakia $(p=0.6)$.

\subsection{The Outcomes of Strabismus after Cataract Surgery}

Using logistical regression analysis, we found that strabismus was more likely to resolve without surgical intervention if the child was older at the time of diagnosis of cataract $(p=0.036)$ and at the time of cataract surgery $(p=0.028)$, however there was no correlation of time to resolution with the duration of cataract or the time from diagnosis to cataract surgery. The average time between date of diagnosis of cataract and time of cataract surgery was 3.1 months $(\mathrm{SD}=1.6)$. For every increase of 1 month of age at diagnosis of cataract, the odds of resolution of the strabismus increased by $2.0 \%$. Logistic regression analysis also showed that, in patients who had resolved strabismus without strabismus surgery, $80 \%$ are older than 14.5 months (95\% CI 63\% - 91\%) and, of those who required strabismus surgery, $65 \%$ are younger than 14.5 months (95\% CI 41\% - 84\%). Using 14.5 months of age as cut off therefore allows us to correctly identify $80 \%$ of the patients whose strabismus would resolve without surgery with a $20 \%$ false positive rate. 
The predicted value of resolution of strabismus is $70 \%$ at an average age of 50 months and increases with age (Figure 1).

The presence of constant strabismus (versus intermittent strabismus) was found to be marginally significant with a p-value of $0.075(\mathrm{OR}=0.314)$ suggesting that intermittent strabismus is more likely to be resolved after cataract surgery only. In this study, we did not find the association between resolution of strabismus and presence of amblyopia or need for amblyopia treatment.

We also assessed the association between resolutions of strabismus and gender, the presence of congenital or acquired cataract, unilateral or bilateral cataract, and duration of cataract, presence of IOL or aphakia. None of these variables showed significance $(p<0.05)$ or marginal significance $(p<0.10)$ in predicting resolution of strabismus after cataract surgery (Table 2).

\section{Discussion}

Strabismus occurs frequently in children with pediatric cataracts and has been described up to $83 \%$ of patients with congenital cataracts [7]. Our study suggests that age is the best predictor of whether the strabismus will resolve without strabismus surgery and that there was a trend towards a higher chance of resolution if the patient had intermittent strabismus. With every increase of 1 month of age at the diagnosis of cataract, the odds for resolution of strabismus increased by $2.0 \%$ in our studied population. Other factors such as amblyopia, type of cataract, and IOl versus aphakia were not found to be significant in predicting resolution of strabismus in our patient population.

Data on the resolution of strabismus in pediatric pa- tients with cataracts are limited in the literature. Weisberg et al. [8] performed a retrospective study of 94 patients (age 18 years or younger) who underwent cataract extraction/intraocular lens implantation (CE/IOL) surgery. Weisberg, et al. found that patients with bilateral cataracts were less likely to develop strabismus than those with unilateral cataracts.

Magli et al. performed a retrospective study of 113 patients with developmental cataracts between the ages of 36 - 144 months in Naples, Italy from 1990 to 2005) [5]. Strabismus was seen in $34 \%$ of their patients before cataract surgery and $41 \%$ after surgery. They found that patients with unilateral cataracts were more likely to have strabismus following cataract surgery $(p=0.002)$ and that $40 \%$ of their patients had amblyopia.

There have been a few studies that have looked at the presence of strabismus in infants who had cataract surgery. In a study by Autrata, et al., strabismus (either esotropia or exotropia) was found in significantly fewer children who received an IOL than those who were left aphakic after cataract surgery; all the patients in this study had surgery before 12 months of age [9]. However, Lambert, et al., in 2001 [9] found that, in 25 infants who had cataract surgery during the first 6 months of life, there was a similar incidence of strabismus between children treated primarily with an IOL and those left aphakic and treated with a contact lens (CL) (75\% vs. $92 \%, p=0.24)$. In Lambert's study, $17 \%$ in the IOL group and $15 \%$ in CL group required strabismus surgery to correct their strabismus. Our study did not find a correlation between resolutions of strabismus with aphakia or pseudophakia, however, the age range in our study is wider as we included older patients. Age of onset of the cataract likely plays a role in the presence and resolution

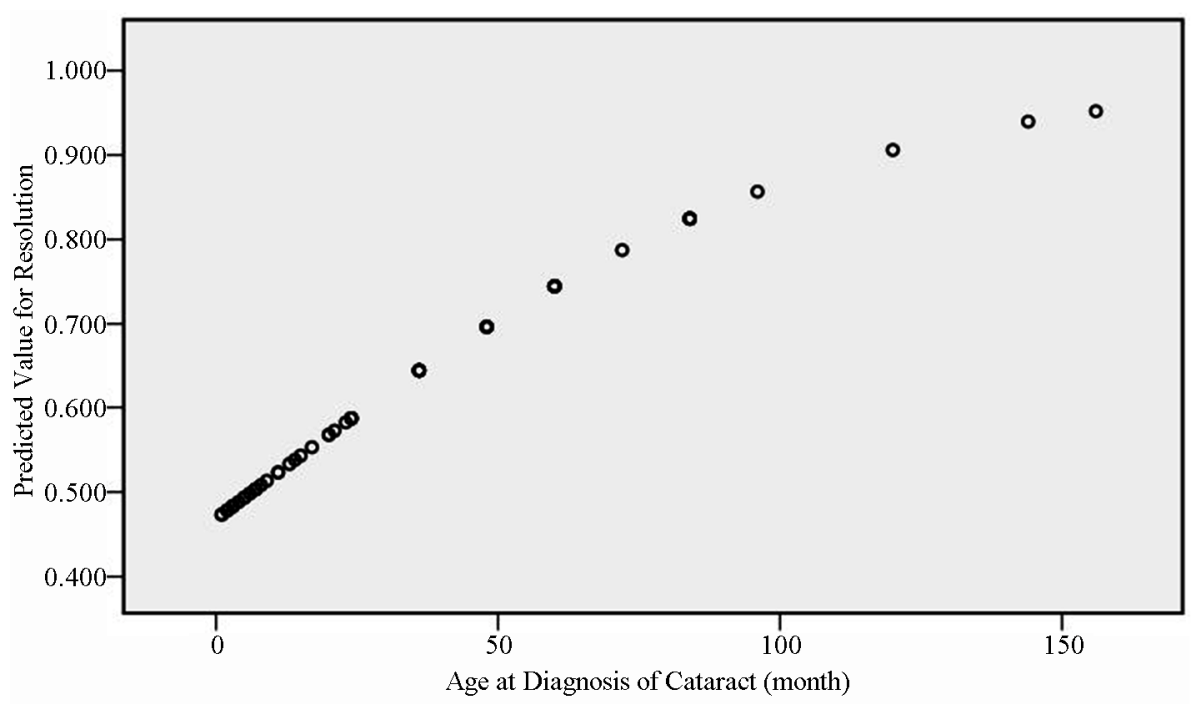

Figure 1. Predicted value for the resolution of strabismus in children with cataract. At age of 50 months old, the average predicted value is $\mathbf{7 0} \%$ and increase with age. 
Table 2. Characteristics of patients with strabismus resolved versus non-resolved cases.

\begin{tabular}{|c|c|c|}
\hline Variables & $\begin{array}{c}\text { Resolved } \\
\mathrm{N}(\%)\end{array}$ & $\begin{array}{c}\text { Non-resolved } \\
\mathrm{N}(\%)\end{array}$ \\
\hline \multicolumn{3}{|l|}{ Gender } \\
\hline Male & $19(56)$ & $11(55)$ \\
\hline Female & $15(44)$ & $9(45)$ \\
\hline \multicolumn{3}{|l|}{ Character of cataract } \\
\hline Congenital & $21(58)$ & $13(72)$ \\
\hline Acquired & $15(42)$ & $5(28)$ \\
\hline \multicolumn{3}{|l|}{ Eye(s) involved } \\
\hline Unilateral & $26(76)$ & $14(70)$ \\
\hline Bilateral & $8(24)$ & $6(30)$ \\
\hline \multicolumn{3}{|l|}{ Types of deviation } \\
\hline Intermittent & $13(42)$ & $10(50)$ \\
\hline Constant & $18(58)$ & $10(50)$ \\
\hline \multicolumn{3}{|l|}{ Amblyopia } \\
\hline Yes & $25(73)$ & $16(80)$ \\
\hline No & $9(26)$ & $4(20)$ \\
\hline \multicolumn{3}{|l|}{ Types of strabismus } \\
\hline ET & $12(38)$ & $11(55)$ \\
\hline XT & $20(62)$ & $9(45)$ \\
\hline \multicolumn{3}{|c|}{ Intraocular lens implantation } \\
\hline Yes & $27(77)$ & $14(70)$ \\
\hline No & $8(23)$ & $6(39)$ \\
\hline
\end{tabular}

ET: esotropia; XT: exotropia.

of strabismus.

Amblyopia was present in $41(76 \%)$ patients. Our results demonstrated that the presence of amblyopia was significantly correlated with younger age and unilateral cataract at surgery, regardless if the patient had pseudophakia or aphakia. However, multiple logistic regression analysis did not find correlation between the resolution of strabismus and the presence of amblyopia ( $73 \%$ versus $80 \%$ ). In Weisberg et al.'s paper, the highest risk of amblyopia was seen in the pseudophakic group with strabismus $(78 \%$ versus $51 \%$ in the pseudophakic non-strabismus group). Weisberg et al. asserted that this might point to an association with the potential for amblyopia in unilateral pseudophakia increasing the possibility of amblyopic strabismus [8].

Based on theories of a latent or "sub-cortical" period in infants, it is estimated that unilateral cataracts which are present beyond 6 weeks can have profound effects on the maturation of the visual pathways with the next sensitive period of cortical plasticity lasting approximately for 7 - 8 years. For bilateral cataracts, Lambert et al. [6] contended that there was no significant advantage for performing cataract surgery before 10 weeks of age, based on their retrospective study of 43 children. Birch et al. [10] argued that there was a linear relationship between the delay in surgery and the long-term visual acuity (VA) outcome for dense bilateral cataracts, with an average loss of one line of VA for every 3 weeks of delay. Accordingly, they contended that surgery during the first four weeks of life was associated with a significantly lower prevalence of strabismus and nystagmus than surgery after 14 weeks.

One topic that we did not address is the change or development of strabismus after extensive patching or amblyopia treatment. Many of pediatric cataract patients undergo extensive occlusion therapy for years after cataract surgery, especially if the cataract is unilateral. In a 1984 study by France and Frank [7], an increase in the incidence of strabismus was found in patients with aphakia after surgery for congenital cataracts with an average follow-up of 4.9 years. This topic would certainly be of importance in future studies.

Our study is limited by the small sample size and retrospective nature of the study. Furthermore, visual acuity and sensory data were not available consistently due to the age of the patients. Variability of strabismus measurements in young children due to cooperation and attention span should also be taken into account. Due to small sample size, we included all pediatric patients with cataracts; studies which assess larger groups of patients at different age ranges and categorize the types and densities of cataracts the patients have would be enlightening.

In summary, we found that age is the best predictor for the resolution of strabismus after cataract surgery and that patients with intermittent strabismus have a trend toward a higher chance of resolution without strabismus surgery. Other factors such as gender, the presence of congenital or acquired cataract, unilateral or bilateral cataract, duration of cataract, and the presence of IOL or aphakia were not found to affect the resolution of strabismus. Future larger studies and randomized trials evaluating the nature and behavior of strabismus in pediatric cataract patients are necessary.

\section{REFERENCES}

[1] C. Gilbert and A. Foster, "Childhood Blindness in the Context of VISION 2020-The Right to Sight," World Health Organization, Vol. 79, No. 3, 2001, pp. 227-232.

[2] A. Foster, C. Gilbert and J. Rahi, "Epidemiology of Cataract in Childhood: A Global Perspective," Journal of Cataract \& Refractive Surgery, Vol. 23, Suppl. 1, 1997, pp. 601-604.

http://dx.doi.org/10.1016/S0886-3350(97)80040-5

[3] U. M. Donnelly, N. M. Stewart and M. Hollinger, "Prevalence and Outcomes of Childhood Visual Disorders," Ophthalmic Epidemiology, Vol. 12, No. 4, 2005, pp. 243250. http://dx.doi.org/10.1080/09286580590967772 
[4] D. S. Friedman, M. X. Repka, J. Katz, L. Giordano, J. Ibironke, P. Hawes, et al., "Prevalence of Decreased Visual Acuity among Preschool-Aged Children in an American Urban Population: The Baltimore Pediatric Eye Disease Study, Methods, and Results," Ophthalmology, Vol. 115 , No. 10, 2008, pp. 1786-1795. http://dx.doi.org/10.1016/j.ophtha.2008.04.006

[5] A. Magli, A. Iovine, D. Bruzzese, U. Giani and F. Fimiani, "Strabismus in Developmental Cataract," European Journal of Ophthalmology, Vol. 18, No. 4, 2008, pp. 540-543.

[6] S. R. Lambert, M. J. Lynn, R. Reeves, D. A. Plager, E. G. Buckley and M. E. Wilson, "Is There a Latent Period for the Surgical Treatment of Children with Dense Bilateral Congenital Cataracts?" Journal of AAPOS, Vol. 10, No. 1, 2006, pp. 30-36.

http://dx.doi.org/10.1016/j.jaapos.2005.10.002

[7] T. D. France and J. W. Frank, "The Association of Strabismus and Aphakia in Children," Journal of Pediatric
Ophthalmology and Strabismus, Vol. 21, No. 6, 1984, pp. 223-226.

[8] O. L. Weisberg, D. T. Sprunger, D. A. Plager, D. E. Neely and N. Sondhi, "Strabismus in Pediatric Pseudophakia," Ophthalmology, Vol. 112, No. 9, 2005, pp. 16251628. http://dx.doi.org/10.1016/j.ophtha.2005.06.002

[9] S. R. Lambert, M. Lynn, C. Drews-Botsch, D. Loupe, D. A. Plager, N. B. Medow, et al., "A Comparison of Grating Visual Acuity, Strabismus, and Reoperation Outcomes among Children with Aphakia and Pseudophakia after Unilateral Cataract Surgery during the First Six Months of Life," Journal of AAPOS, Vol. 5, No. 2, 2001, pp. 70-75. http://dx.doi.org/10.1067/mpa.2001.111015

[10] E. E. Birch, C. Cheng, D. R. Stager Jr., D. R. Weakley Jr. and D. R. Stager Sr., "The Critical Period for Surgical Treatment of Dense Congenital Bilateral Cataracts," Journal of AAPOS, Vol. 13, No. 1, 2009, pp. 67-71. http://dx.doi.org/10.1016/j.jaapos.2008.07.010 